\title{
Review of: "Crossover patterning through kinase- regulated condensation and coarsening of recombination nodules"
}

\author{
Akira Shinohara ${ }^{1}$ \\ 1 Osaka University
}

Potential competing interests: The author(s) declared that no potential competing interests exist.

Review:

Title: Crossover patterning through kinase-regulated condensation and coarsening of recombination nodules

Authors: Liangyu Zhang, Weston Stauffer, David Zwicker, Abby F. Dernburg

The paper by Zhang et al. describes the characterization of a meiosis-specific CDK complex in C. elegans, CDK-2/COSA-1 and CDK-2/COSA-1-dependent phosphorylation of MSH-5, which is a component of MutSgamma complex, in meiotic crossover formation. Moreover, the paper described a new model on the development of recombination complexes as biomolecular condensates along chromosomes (a model for crossover interference). The authors showed, by using the auxin degron system, C. elegans CDK-2, is essential for the formation of recombination nodules (RN) essential for crossover formation during meiosis CDK-2 is also essential for proliferation of germ line stem cells. The authors identified possible CDK-2 phosphorylation sites in the C-terminal region of Msh5 and the substitution of several putative residues as non-phosphorylated forms decreased formation of MSH-5 focus as well as ZHP-3 (instead line-staining). The substitution into phospho-mimetic version induced aggregate formation of the mutant MSH-5 protein. The authors also measured dynamics of different RN components and showed ZHP-3/-4 shows more mobile like synaptonemal complex (SC) components, SYP-1 and SYP-3 etc, while late RN components such as MSH-5m CDK-2, and COSA-1 are immobile. Based these observations, the authors proposed a model in which RN has a property of biomolecular condensates accompanied with communication along SCs.

The works are designed well and technically executed in a proper way. And the model using biomolecular condensates sounds interesting, which is similar to the model proposed by Morgan et al. (Nature Communs, 2021; DOI: 10.1038/s41467-021-24827-w) as noted by the authors. However, because of concerns listed below, the authors need to clarify the experimental results with non-biased interpretation. Before the publication, the paper may need some additional works to support the authors' interesting claims. 
Major points;

1. Although the authors showed the substitution of a half of 13 putative CDK sites in the C-terminal region of MSH-5; MSH-5 ${ }^{\mathrm{NP1}}$ (5 alanine substitutions) or $\mathrm{MSH}-5^{\mathrm{NP2}}$ (6 alanine substitutions), abolished the focus formation of MSH-5 (Figure 3B), a recent BioRxiv paper by Haversat et al.

(DOI: 10.1101/2021.08.31.458431) showed the substitution of all 13 sites in MSH-5 does not show any meiotic defects in wild-type worm background, which is inconsistent with the results described here. One clear difference is in the tag used for each experiment; in this paper, N-terminal GFP fusion is used while in the paper by Haversat used C-terminal V5 tagged version. The authors need to dissolve this discrepancy. Particularly, the use of GFP-tagged MSH-5 does not look good. Since the role of CDK phosphorylation sites in MSH-5, which is a major likely target of CDK-1/COSA-1, is a key finding in the paper. The authors need to clarify this issue.

2. The authors claim that CDK-dependent phosphorylation of the C-terminal region of MSH-5 promotes aggregation formation of MSH-5 (and other interacting proteins). There is few evidence supporting this. Although MSH-5 ${ }^{\mathrm{PM} 2}$ protein is defective in focus-formation, but forms unusual aggregates outside of a cell (no aggregates "in" a cell, Figure $3 \mathrm{C}$ ), $\mathrm{MSH}-5^{\mathrm{PM} 1}$ protein does not form a clear aggregate by midpachytene stage, rather showed normal focus formation (Figure 3C).

3. In the same line to \#2, it is better for the authors to show early MSH-5 foci are different from late foci, in terms of amounts of proteins (intensity), dynamics, and/or amounts of phosphorylation. Furthermore, the model (Figure 7) shows the multimer formation of CDK-2/COSA-1 (and ZHP-3/-4) by the phosphorylation, not MSH-5/MSH-4 (Him-16). Is there any data to support this? If not, multimer formation of MSH-5/MSH-4 should be included in the model.

4. FRAP assay in Figure 5: If the phosphorylation of MSH-5 recruits more CDK2/COSA-1 in LN as suggested in this paper (Figure 7), the authors would see signal recovery of LN foci, particularly CDK-2/COSA-1 (or MSH-5) in FRAP. However, this is not the case for CDK-2 and MSH-5 foci (Figure 5B). Again, this does look to support the idea of dynamic nature of RN. The brightness of RM/LM foci are quite heterologous (Figure 5A). It is important to check both bright and less bright foci show similar FRAP kinetics. Moreover, meiotic chromosomes in C. elegans move. How did the author compensate the effect of the motion on the assay (the same for FCS).

5. The authors concluded that RN contains "highly" dynamic components (page 9, line 7 from the bottom). ZHP-3/-4 foci are not highly dynamics, since FRAP showed partial recovery of the fluorescence (Figure 5B). It would be better to do FRAP for ZHP-3 in CDK-2 depletion or COSA-1 mutants, which shows line-like localization of ZHP-3. This is a good support for the authors' model (Figure 7).

6. Page 11, last paragraph, Figure $7 \mathrm{C}, \mathrm{D}$, and E; Please explain more in detail in the main text.

Minor points;

1. Page 4, line 4 from the bottom; "p.2" should be removed.

2. Figure $1 \mathrm{~A}$; For the readers not familiar with $\mathrm{C}$. elegance meiosis, please show which region of the gonad 
is mid-pachytene and diplotene etc.

3. Page 5, third paragraph, line 5; (data not shown). What kind of data did the authors mention? Please show the data in Supplemental Figure.

4. Figure 2A: Please show images with ZHP-3 alone including early time points.

5. Page 7, first paragraph, line 10; (data not shown). Please show the data of CDK-2 phosphorylation in Supplemental Figure.

6. Page 7, second line from the bottom; Please remove "8/26/21 7:17:00 PM".

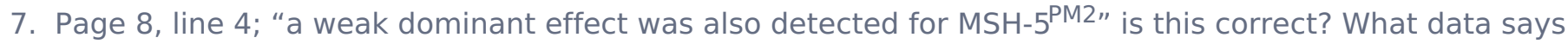
this? If so, this is significant?

8. What is the MSH-5 localization in heterozygous mutant for $\mathrm{MSH}-5^{\mathrm{PM} 1}$ or $\mathrm{MSH}-5^{\mathrm{PM} 2}$ ? Do those heterozygotes show normal MSH-5 foci or aggregates?

9. In Figure 4, what does WT/+ mean? Probably, GFP::MSH-5/+. If so, please show exact phenotypes in the label.

10. Figure $4 \mathrm{D}$, what happens to $\mathrm{ZHP}-3$ foci in $\mathrm{MSH}-5^{\mathrm{NP1}}$ or $\mathrm{MSH}-5^{\mathrm{NP} 2}$, which are defective in $\mathrm{MSH}-5$ focus formation? These are more important than those for $\mathrm{MSH}-5^{\mathrm{PM} 1}$ or $\mathrm{MSH}-5^{\mathrm{PM} 2}$.

11. Figure 5B: FRAP analysis of RPA-1 foci is presented. However, there are less words on the nature of RPA1 in RN and its function in the text. 